\section{rev Psi}

Revista de Psicología (UNLP)

https://revistas.unlp.edu.ar/revpsi

Artículo de investigación

\title{
Predicción de actitud hacia personas con VIH/SIDA y hacerse prueba del VIH
}

José Moral de la Rubia

Adrián Valle de la $0^{2}$

\section{Correspondencia}

jose_moral@hotmail.com

Filiaciones institucionales

${ }^{1}$ Facultad de Psicología, Universidad

Autónoma de Nuevo León (México)
${ }^{2}$ Tecnológico de Monterrey (México)

\section{Resumen}

Esta investigación tiene como objetivo contrastar un modelo para predecir actitud hacia personas que viven con VIH/SIDA (PVVS) y hacerse la prueba del VIH. Se aplicó la Escala de Actitud hacia PVVS, la escala de actitud hacia hombres homosexuales y lesbianas, la escala de autoritarismo de ala derecha y preguntas sobre datos sociodemográficos, clínicos, de conducta sexual y relaciones de amistad a 202 estudiantes de medicina mexicanos. Se usó un muestreo no probabilístico. Con buen ajuste a los datos, una actitud de mayor rechazo hacia PVVS fue predicha por orientación heterosexual, ser hombre y sumisión autoritaria. La actitud hacia personas homosexuales tuvo una correlación sustantiva con actitud hacia PVVS, pero no fue un predictor. Hacerse la prueba del VIH fue predicho por ser hombre, actitud de aceptación hacia PVVS, tener amigos homosexuales y sumisión autoritaria. Se concluye que el modelo especificado de corte psicosocial presenta gran capacidad explicativa.

\section{Palabras clave}

actitud | homosexualidad | autoritarismo | estudiantes | México

\section{Cómo citar}

Moral de la Rubia, J. y Valle de la O,

A. (2020). Predicción de actitud hacia personas con VIH/SIDA y hacerse prueba del VIH. Revista de Psicología, 19(1), 157178. doi: $10.24215 / 2422572 \mathrm{XE0} 66$
Recibido

17 nov. 2019

\section{Aceptado}

10 may. 2020

Publicado

17 may. 2020

Editor

Nicolás Alessandroni | Facultad de Psicología, Universidad Autónoma de Madrid (España)
ISSN

2422-572X

Licencia

(c) Copyright: Moral de la Rubia, J. y Valle de la O, A. Licencia de Cultura Libre CC-BY 4.0

Entidad editora

RevPsi es una publicación de la

Facultad de Psicología (Universidad Nacional de La Plata, Argentina)

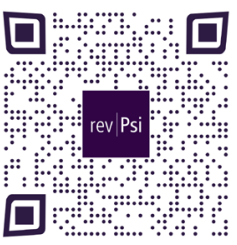

ACCESO ABIERTO DIAMANTE 2 


\section{Previsão de atitude em relação às pessoas com HIVIAIDS e testagem para HIV}

\section{Resumo}

Esta pesquisa tem como objetivo testar um modelo para prever atitudes em relação às pessoas que vivem com HIV/AIDS (PVHA) e fazer o teste para o HIV. A Escala de Atitude em relação às PVHA, a escala de atitude em relação a homens homossexuais e lésbicas, a escala de autoritarismo da direita e perguntas sobre relações sociodemográficas, clínicas, comportamento sexual e relações de amizade foram aplicadas a 202 estudantes de medicina mexicanos. Amostragem não probabilística foi utilizada. Com um bom ajuste aos dados, foi prevista uma atitude de maior rejeição em relação às PVHA por orientação heterossexual, ser homem e submissão autoritária. A atitude em relação às pessoas homossexuais teve uma correlação substantiva com uma atitude em relação às PVHA, mas não foi um preditor. Fazer o teste para o HIV foi previsto por ser homem, uma atitude de aceitação em relação às PVHS, ter amigos homossexuais e submissão autoritária. Conclui-se que o modelo especificado de corte psicossocial apresenta grande capacidade explicativa.

\section{Palavras-chave}

atitude | homossexualidade | autoritarismo | estudantes | México

\section{Prediction of attitude toward people with HIVIAIDS and get- ting tested for HIV}

\section{Abstract}

This research aims to test a model to predict the attitude toward PLWHA and getting tested for HIV. The Scale of Attitude toward, the Scale toward Lesbians and Gays, the scale of Right Wing Authoritarianism and questions about sociodemographic information, clinical data, sexual behaviour and relationships of friendship were applied to 202 Mexican medical students. A non-probability sampling was used. With a close data fit, an attitude of greater rejection towards PLWHA was predicted by heterosexual orientation, being man, and authoritarian submission. The attitude toward homosexual persons had a substantive correlation with attitude toward PLWHA, but it was not a predictor. Getting tested for HIV was predicted to be a man, an attitude of acceptance towards PLWHA, having homosexual friends, and authoritarian submission. It is concluded that the specified model of psychosocial perspective has a great explanatory capacity.

\section{Keywords}

attitude | homosexuality | authoritarism | students | Mexico 


\section{Aspectos destacados del trabajo}

- Entre estos estudiantes médicos mexicanos de universidad privada, los aspectos de agresión autoritaria no poseen fiabilidad.

- El autoritarismo se reduce a un factor de sumisión a autoridades y normas sociales tradicionales.

- Orientación heterosexual, ser hombre y sumisión autoritaria predicen rechazo hacia personas que viven con VIH/SIDA (PVVS).

- Ser hombre, aceptar PVVS, tener amigos homosexuales y sumisión autoritaria predicen hacerse la prueba del VIH.

La presencia de una actitud negativa hacia personas que viven con virus de inmunodeficiencia humana y síndrome inmunodeficiencia adquirida (PVVS) en el ámbito clínico puede tener un efecto deletéreo en la atención hacia dicha población (Geter, Herron y Sutton, 2018), de ahí la importancia de identificar las variables que permitan predecir o explicar una actitud de rechazo hacia PVVS con las que poder diseñar intervenciones de cambio de actitud y evaluar entornos de atención clínica, especialmente a nivel de estudiantes de pregrado. Por otra parte, considerando el fuerte consenso médico en la importancia de hacerse la prueba del virus de inmunodeficiencia humana (VIH) para una detección y tratamiento oportuno de la infección (Kalichman, 2015), es de gran interés conocer los predictores de esta conducta de salud entre jóvenes universitarios.

La presente investigación propone y contrasta un modelo predictivo enmarcado dentro de una perspectiva psicosocial (Herek, 2009). Este enfoque teórico neofuncional plantea que las actitudes tienen funciones adaptativas, expresivas y defensivas intrapsíquicas. Además, sostiene que el nivel actitudinal de un individuo viene determinado por características sociodemográficas, grupos de pertenencia e identidad, experiencias con el objeto actitudinal, otras actitudes hacia objetos relacionados, rasgos de personalidad y el contexto socio-cultural (Herek, 2009).

Tras la revisión de investigaciones sobre actitud hacia PVVS y hacerse la prueba del VIH centradas en variables psicosociales y publicadas durante las dos últimas décadas, siete variables destacaron como correlatos o predictores significativos. Retomando las mismas, el modelo propuesto para predecir actitud hacia PVVS y hacerse la prueba del VIH incluye el sexo como predictor sociodemográfico (Dahlui et al., 2015; Kikwasi, Lukwale y Mwageni, 2017; Masoudnia, 2015; Tang et al., 2016), la orientación sexual (Boone et al., 2016) y tener amigos homosexuales (Burke et al., 2015; Knaak y Patten, 2016; Prati et al., 2016) como predictores relacionados con los grupos de identidad o pertenencia y experiencias vividas, la actitud hacia personas homosexuales (Suominen et al., 2015; Vaughn, Teeters, Sadler y Cronan, 2017) como otra actitud hacia un objeto relacionado, la religiosidad (Reyes, Varas y Martínez, 
2015) como predictor ideológico (desde valores cristianos católicos o no católicos) y el autoritarismo de ala derecha (Rickles, Furtek, Malladi, Ng y Zhou, 2016) como predictor ideológico (de derechas) y de rasgo (rigidez autoritaria).

Desde un modelo de conocimiento/actitud (Farhat, Greene, Paige, Koblin y Frye, 2017), hacerse la prueba del VIH puede ser predicho desde el conocimiento que se tiene de la infección, la práctica de conductas sexuales de riesgo y la actitud hacia la prueba o ser seropositivo (PVVS). Al hacerse la presente investigación en estudiantes de medicina, el conocimiento se consideró una variable constante (con valor techo), por lo que no iba a permitir explicar la varianza de la conducta de salud y se obvió. En el cuestionario aplicado enfocado a actitudes, el número de parejas sexuales fue la única conducta sexual de riesgo medida, por lo que se contempló como un posible predictor (Huerga et al., 2016). Orientación no heterosexual (Huerga et al., 2016; Jin et al., 2002), ser hombre (Asaolu et al., 2016) y tener amigos homosexuales (Wesolowski et al., 2019) pueden actuar de forma directa desde su asociación con conductas sexuales de riesgo o de forma indirecta por mediación de la actitud hacia PVVS. La actitud hacia personas homosexuales (Mukherjee, McKinney y Darrow, 2018), religiosidad (Reyes et al., 2015) y autoritarismo de ala derecha (Rickles et al., 2016) pueden actuar de forma indirecta por mediación de la actitud hacia PVVS. A su vez, el autoritarismo de ala derecha puede actuar de forma directa como rasgo o tendencia a ver al otro como peligroso (parejas sexuales) y acatar ciegamente los preceptos y órdenes de las autoridades (Ungaretti, Müller y Etchezahar, 2016), como hacerse la prueba preventivamente ante la pandemia actual (Kalichman, 2015).

El objetivo de este estudio es comprobar un modelo predictivo de actitud hacia PVVS y hacerse la prueba del VIH entre estudiantes de medicina. Desde una perspectiva psicosocial y una teoría neofuncional de la actitud (Herek, 2009), se consideran variables sociodemográficas (sexo y edad), religiosidad, variables de conducta sexual (orientación sexual, vida sexual activa y número de parejas sexuales), contacto personal con el objeto estigmatizado (relaciones de amistad con personas homosexuales y PVVS y haber tratado a PVVS), actitud hacia objetos relacionados (personas homosexuales) y una variable de rasgo (autoritarismo de ala derecha).

Al especificar el modelo, se espera que la actitud de rechazo hacia PVVS sea predicha por ser hombre (Dahlui et al., 2015; Kikwasi et al., 2017; Masoudnia, 2015; Tang et al., 2016), orientación heterosexual (Boone et al., 2016), no tener amigos homosexuales (Burke et al., 2015; Knaak y Patten, 2016; Prati et al., 2016), actitud de rechazo hacia personas homosexuales (Emlet et al., 2015) y mayor autoritarismo de ala derecha (Burgess et al., 2019; Rickles et al., 2016). Tener amistad con PVVS probablemente sea una variable problemática al aproximarse a una constante $(0=$ No), debido a que es una enfermedad de muy baja prevalencia poblacional. No se espera que número de parejas sexuales y vida sexual activa jueguen papeles relevantes, al igual que ocurre al predecir actitud hacia personas homosexuales en esta población de estudiantes (Moral y Valle, 2011a).

Por otra parte, se espera que hacerse la prueba del VIH sea predicho directamente por una actitud de mayor aceptación hacia PVVS (Weiser et al., 2006) e indirectamente 
por los predictores previamente mencionados de esta actitud. Se espera que ser hombre (Asaolu et al., 2016), orientación no heterosexual (Huerga et al., 2016; Jin et al., 2002) y tener amigos no heterosexuales (Wesolowski et al., 2019) en relación con conductas sexuales de riesgo, así como mayor autoritarismo desde la obediencia a la autoridad (acatar la consigna contra el negacionismo del VIH/SIDA) predigan directamente hacerse la prueba del VIH. Otras dos variables que podrían jugar un papel importante como predictores directos de hacerse la prueba del VIH son el número de parejas sexuales (Huerga et al., 2016) o ser sexualmente activo (DanielUlloa et al., 2016) en relación con las conductas sexuales de riesgo.

Las variables tratar a PVVS y edad probablemente no sean relevantes para el modelo por correlaciones no significativas debido a su limitada variabilidad en la muestra de estudiantes de segundo y tercer año de carrera. Finalmente, la religiosidad es una variable compleja desde el conflicto que se plantea entre los valores de aceptación hacia los enfermos y excluidos y los valores de rectitud moral (Reyes et al., 2015), por lo que probablemente no tenga un efecto relevante para el modelo.

Al presentar el modelo seis o cinco variables exógenas con $15 \mathrm{o} 10$ posibles correlaciones, se consideró la posibilidad de especificar dos de ellas como endógenas: actitud hacia personas homosexuales y tener amigos homosexuales. Se espera que mayor rechazo hacia personas homosexuales sea predicho por ser hombre, ser heterosexual y mayor autoritarismo de ala derecha (Herek, 2009; Moral y Valle, 2019); a su vez, tener amigos homosexuales sea predicho por ser no heterosexual, ser mujer, menor autoritarismo y actitud de menor rechazo hacia las personas homosexuales (Hodson, Turner y Choma, 2016).

\section{Método}

\section{Diseño}

Esta investigación cuantitativa de tipo correlacional usó un diseño ex post facto de corte transversal.

\section{Participantes}

Se usó un muestreo no probabilístico. Se reclutó una muestra incidental de 202 participantes voluntarios en una facultad de medicina de una universidad privada de Monterrey, México. El criterio de inclusión fue ser estudiante de medicina. El criterio de exclusión fue no conceder el consentimiento informado. No se usó ningún método de sustitución de los valores perdidos. Todos los alumnos a los que se les invitó a formar parte de la investigación dieron su consentimiento informado. La aplicación del cuestionario fue colectiva en salones de clase correspondiente a segundo y tercer año de licenciatura. La colecta de los datos fue realizada entre los meses de agosto a diciembre de 2017.

Al momento de determinar el tamaño de la muestra, se consideró el contraste de la significación de una correlación múltiple en regresión lineal y análisis de senderos. 
Con cinco variables predictoras con un tamaño del efecto grande sobre la variable predicha (hipótesis alternativa: $\mathrm{H}_{1}=25 \%$ ) y una potencia de 99 , se requieren al menos 86 participantes. Si se sube a seis variables predictoras, el mínimo es de 91 participantes y si se sube a siete es de 96 . Para usar técnicas de análisis de senderos, se indica un tamaño de muestra mínimo de 100 participantes y al menos cinco participantes por parámetro a estimar (Kyriazos, 2018), lo que se alcanzó en la submuestra en la que se hizo este contraste, 130:26 $=5: 1$ en el modelo inicial y 130:21 $=6,2: 1$ en el modelo final. El porcentaje de muestreo fue de 21,9\% en la población de 923 estudiantes de medicina.

En la muestra de 199 participantes analizada (eliminados tres casos por casos incompletos en la escala de actitud hacia PVVS), 51,1\% eran mujeres y $48,9 \%$ hombres. El porcentaje de ambos sexos fue estadísticamente equivalente por la prueba binomial, $p=, 828$, y equivalente al de la población de estudiantes de medicina de la universidad privada en la que se recolectaron los datos, $\chi 2(1, N=190)=0,17, p=, 684$. Todos ellos dijeron ser solteros. La media de la edad fue 19,81 con una desviación típica de 1,16 y un rango de 9 (entre 17 y 26). Su distribución mostró ligera asimetría positiva y fuerte apuntamiento, debido a que las edades se concentraron en los valores de 20 y 19, ya que predominaban alumnos de segundo y tercer año de licenciatura.

El 79\% indicó ser cristiano católico, $8 \%$ cristiano no católico, 10,5\% ateo o agnóstico, $1,5 \%$ creyente sin religión definida y $1 \%$ budista. En un rango de 0 "nunca" a 12 " 6 o 7 días a la semana", la religiosidad o frecuencia de asistencia a servicios religiosos varió de 0 a 9 ("de 4 a 5 veces por semana"). Su mediana correspondió a 5 "una vez al mes" y su moda fue 7 "una vez a la semana”. Su distribución se sesgó hacia valores de baja frecuencia de asistencia a servicios religiosos.

Entre los 191 participantes que respondieron a la pregunta si han tenido relaciones sexuales, $48,7 \%$ dijo que sí y 51,3\% no. Ambos porcentajes fueron estadísticamente equivalentes por la prueba binomial, $p=, 772$. De los 198 estudiantes que respondieron a la pregunta sobre su orientación sexual, $94,9 \%$ indicaron ser heterosexuales y 5,1\% no heterosexuales, siendo $3,6 \%$ bisexuales y $1,5 \%$ homosexuales.

Entre los 169 participantes que respondieron a la pregunta sobre el número de parejas sexuales en los últimos seis meses, 57,4\% respondió que no tuvo ninguna, 34,9\% una, $5,9 \%$ dos, $1,2 \%$ tres y $0,6 \%$ nueve. La media del número de parejas fue 0,56 y la desviación típica fue 0,93 . La distribución mostró asimetría positiva y leptocurtosis. Con respecto a la prueba del VIH, 83,9\% se habían hecho la prueba. En sus prácticas clínicas, 10,1\% de los estudiantes reportaron haber atendido a PVVS. Con respecto a relaciones de amistad, $77 \%$ reportaron tener amigos homosexuales y 3,6\% tener amigos que viven con VIH.

\section{Instrumentos de medida}

Se aplicó un cuestionario de autorreporte. Este iniciaba con preguntas cerradas sobre el sexo (hombre o mujer), orientación sexual (heterosexual, bisexual u homosexual), tener relaciones de amistad con PVVS y personas homosexuales, vida sexual activa, hacerse la prueba del VIH y haber tratado a PVVS (sí o no), adscripción religiosa 
(cristiana católica, cristiana no católica, ateo/agnóstico y otra) y religiosidad (frecuencia de asistencia a servicios religiosos). El cuestionario también incluía preguntas abiertas sobre la edad y el número de parejas sexuales en los últimos seis meses.

Tras estas preguntas aparecían tres escalas, dos de actitud y una de autoritarismo. Las escalas estaban conformadas por ítems con un formato de respuesta cerrado con cinco categorías ordenadas. Se usaron los valores 1-3-5-7-9 en lugar de 1-23-4-5 para puntuar las cinco categorías de respuesta a los ítems, al constituir una transformación lineal admisible que puede facilitar el ajuste a la normalidad (Bishop $y$ Herron, 2015). La puntuación en cada escala se obtuvo sumando los valores de los ítems (puntuados en sentido de actitud de rechazo o posicionamiento ideológico autoritario) y dividiendo por el número de ítems sumados, por lo que el rango varía en un intervalo continuo de 1 a 9. En términos absolutos, una puntuación menor que 4,2 muestra desacuerdo con las afirmaciones de rechazo o autoritarias (actitud de aceptación o posicionamiento liberal), entre 4,2 y 5,79 refleja un posicionamiento ambiguo y una puntuación mayor o igual que 5,8 indica acuerdo con las afirmaciones de rechazo (actitud de rechazo o posicionamiento autoritario).

Escala de Actitud hacia Personas que Viven con VIH/SIDA (EA-PVVS-6; Moral y Valle, en prensa). Consta de seis ítems con un formato de respuesta cerrado con cinco categorías ordenadas de desacuerdo. Resulta de la adaptación mexicana de la escala de Neumann et al. (2004) tras eliminar un ítem por falta de confiabilidad. La consistencia interna de la EA-PVVS- 6 fue aceptable, $\alpha=, 71$, y presentó una estructura unifactorial con buen ajuste a los datos (Moral y Valle, en prensa). Una mayor puntuación en la escala refleja una actitud de mayor rechazo hacia PVVS.

Escala de Actitud hacia Lesbianas y Hombres Homosexuales (ATLG). Fue creada por Herek (1984) y adaptada en México por Moral y Valle (2011b). Consta de 20 ítems con una escala de respuesta tipo Likert de cinco puntos en la que los ítems se puntúan de 1 a 9. Mayor puntuación refleja una actitud de mayor rechazo hacia las personas homosexuales. Moral y Valle (2013) concluyeron que la estructura de la escala es unidimensional con un ajuste aceptable a los datos y equivalente a los modelos de dos y tres factores que mostraron falta de validez discriminante. Su factor presentó validez convergente y consistencia interna excelente, $\alpha=, 91$.

La escala de autoritarismo de ala derecha (RWA). Se aplicó la versión de 12 ítems (RWA-12) de Cárdenas y Parra (2010) más un ítem adicional. El ítem añadido fue extraído del estudio sobre la homonegatividad en el ejército de Herek (1996): "a una persona abiertamente homosexual se le debería permitir dar servicio en el ejército". Consta de seis ítems redactados en sentido autoritario (ítems $1,3,5,8,9$ y 12) y siete en sentido liberal (ítems 2, 4, 6, 7, 10, 11 y 13). En la RWA-12, los ítems se puntúan en sentido de mayor grado de acuerdo con afirmaciones autoritarias y mayor grado de desacuerdo con las liberales, de tal forma que una mayor puntuación en la escala muestra una actitud más autoritaria. No está validada en México. En el estudio realizado en Chile por Cárdenas y Parra (2010) mostró una estructura de tres factores: agresión autoritaria (ítems 3, 5, 9, 11 y 12), sumisión autoritaria (ítems 
1, 2,4 y 6) y convencionalismo (ítems 7,8 y 10). La consistencia interna global fue aceptable $(\alpha=, 72)$, pero no se reportó la de los factores.

\section{Procedimiento}

Se obtuvo el permiso y aprobación de las autoridades académicas del departamento de la universidad privada en la que se colectaron los datos, aunque el proyecto no se sometió a ningún comité de ética. Se solicitó el consentimiento informado de los estudiantes para su participación en la investigación. Este aparecía en la primera hoja del cuestionario. No se pidió ningún dato de identificación personal para garantizar el anonimato de las respuestas. Se proporcionó el nombre y la dirección de correo electrónico de los responsables de la investigación para solicitar información en relación con cualquier duda suscitada por este estudio. De este modo, se cumplió con las normas éticas de investigación de la American Psychological Association (2017).

\section{Análisis de datos}

Se empleó un muestreo no probabilístico. Esta técnica posee menos garantías de proporcionar una muestra aleatoria o de casos independientes que el muestreo probabilístico (Efron y Hastie, 2016). Al ser la aleatoriedad un supuesto básico de toda prueba de inferencia estadística, se contrastó la aleatoriedad de las series de datos de las variables analizadas por la prueba de rachas de Wald y Wolfowitz (Cui, Li, Yang y Xu, 2018). Las secuencias se dispusieron en su orden de colecta. Los contrastes fueron bilaterales con un nivel de significación de, 05 . Los cálculos se hicieron con SPSS24, módulo R4.3 para SPSS24, Excel2013, AMOS16 y LISREL8.5.

Las correlaciones con la EA-PVVS-6 se calcularon por correlaciones heterogéneas. Con variables cualitativas dicotómicas y ordinales, se usó el coeficiente poliserial. Su significación se contrastó por la prueba de Wald y el supuesto de normalidad bivariada por la prueba chi-cuadrado y el error de aproximación cuadrático medio (RMSEA). Una $p>, 05$ para la prueba chi-cuadrado y una $p>, 10$ para la hipótesis nula de RMSEA $<, 05$, se tomaron como criterios de buen ajuste al modelo de distribución normal bivariada. Con las variables cuantitativas, se usó el coeficiente de correlación producto-momento de Pearson. La normalidad univariada se comprobó por la prueba de D’Agostino y Pearson (basado en las medidas de asimetría y curtosis de muestra de Fisher) y la bivariada por el estadístico $U$ de asimetría multivariada y el estadístico W de curtosis multivariada. En caso de incumplimiento, se estimó el intervalo por muestreo repetitivo simple con la simulación de 2000 muestras aleatorias a través del método de percentiles.

Se definió un modelo estructural por análisis de senderos. La función de discrepancia se optimizó por Máxima Verosimilitud desde la matriz de correlaciones heterogéneas y la estimación por intervalo de los parámetros se hizo por percentiles corregidos de sesgo con la simulación de 2000 muestras aleatorias. La bondad de ajuste se valoró por medio de ocho índices: prueba chi-cuadrada ( $p$ de $\chi 2$ ), probabilidad de Bollen y Stine por muestreo repetitivo con la simulación de 2000 muestras aleatorias ( $p$ de Bollen y 
Stine), índice de bondad de ajuste de Jöreskog y Sörbom (GFI) y su variante ajustada (AGFI), índices normado de ajuste (NFI), índice comparativo de ajuste (CFI), error de aproximación cuadrático medio (RMSEA) y residuo cuadrático medio (SRMR).

Siguiendo a Byrne (2016), valores de $p$ de $\chi 2>, 05, p$ de Bollen y Stine $>, 05$, GFI, NFI y $C F I \geq, 95, A G F I \geq, 90$ y $R M S E A$ y $S R M R \leq, 05$ se consideraron que reflejan un buen ajuste; $\mathrm{y}$ valores de $\mathrm{p}$ de $\chi^{2}>, 01, p$ de Bollen y Stine $>, 01$, GFI, NFI y CFI $\geq, 90, A G F I$ $\geq, 85, R M S E A \leq, 075$ y $S R M R<, 10$ se juzgaron que muestran un ajuste aceptable. Se interpretó que valores absolutos en los coeficientes de correlación o pesos estructurales estandarizados entre 10 y, 29 reflejan una fuerza de asociación o un tamaño del efecto pequeño, entre, 30 y, 49 medio, entre, 50 y , 69 grande $y \geq, 70$ muy grande (Byrne, 2016).

Cabe señalar que el análisis de senderos nació de la necesidad de proporcionar una mayor flexibilidad a los modelos de regresión múltiple. No es una técnica meramente confirmatoria, sino que puede usarse tanto para poner a prueba un modelo hipotético como para modificarlo desde los datos muestrales y proponer nuevos modelos con base en la eliminación de vías sin pesos estructurales significativos y las indicaciones de los índices de modificación con sentido teórico (Byrne, 2016), tal como se hace en este estudio. Por otra parte, el análisis de senderos requiere un tamaño de muestra más pequeño que el modelamiento de ecuaciones estructurales con variables latentes para alcanzar la misma potencia (Kyriazos, 2018). A su vez, permite mantener todos los ítems o contenidos que definen una variable sin necesidad de reducir el modelo de medida (variable latente o no observada) a 3 o 4 indicadores para lograr un buen ajuste a los datos, como suele ocurrir en el modelamiento de ecuaciones estructurales.

Al usarse análisis de senderos, las variables actitud hacia PVVS (medida con seis ítems de la EA-PVVS-6), autoritarismo de ala derecha (medido con seis ítems de la RWA) y actitud hacia personas homosexuales (medida con 20 ítems de la ATLG) se incluyeron como variables manifiestas (observadas) en el modelo. No obstante, se validó un modelo de medida unidimensional (de un factor) para cada una de ellas, se comprobó la validez convergente del factor y se estimó su fiabilidad de consistencia interna. La estimación puntual de parámetros en el modelo de un factor se hizo por mínimos cuadrados libres de escala desde la matriz de correlaciones policóricas y la estimación por intervalo se computó por percentiles corregidos de sesgo, al tratarse de variables ordinales (Byrne, 2016).

Se define validez convergente como el grado de certeza en que los ítems que integran un modelo de medida definen un mismo constructo. Esta propiedad se comprobó a través de los pesos de medida estandarizados $(\lambda)$, la varianza media extraída $(A V E)$ y la confiabilidad compuesta ( $\omega$ ). Con seis ítems, valores de $\lambda \geq, 50, A V E \geq, 40$ y $\omega \geq$ , 80 muestran una validez convergente buena y $\lambda \geq, 50, A V E \geq, 28$ y $\omega \geq, 70$ aceptable. Con 20 ítems, valores de $\lambda>, 50, A V E \geq, 31$ y $\omega \geq, 90$ reflejan una validez convergente buena y $\lambda \geq, 50, A V E \geq, 25$ y $\omega \geq, 87$ aceptable (Moral, 2019).

La fiabilidad de consistencia interna se refiere al grado de exactitud que tiene el test para medir el constructo. Se estima desde la covarianza o correlación entre los ítems que lo integran. Esta propiedad se calculó a través el coeficiente alfa ordinal ( $\alpha$ 
ordinal). Este coeficiente se basa en la media de las correlaciones policóricas entre los ítems; un valor de $\alpha$ ordinal $<, 50$ se suele interpretar como un nivel de confiabilidad inaceptable, de , 50 a ,59 muy bajo, de ,60 a ,69 cuestionable, de ,70 a ,79 aceptable, de , 80 a ,89 bueno y de .90 a 1 excelente (Espinoza y Novoa-Muñoz, 2018).

\section{Resultados}

Al disponer los casos en el orden en que los cuestionarios fueron entregados al encuestador por los participantes, se mantuvieron las hipótesis nulas de aleatoriedad en las secuencias de datos de las 26 variables analizadas por la prueba de rachas. Por tanto, se pudo asumir el supuesto de aleatoriedad requerido para el uso de estadística inferencial.

\section{Confiabilidad, distribución y tendencia central de las escalas psicométricas}

La consistencia interna de la EA-PVVS-6 fue aceptable, $\alpha$ ordinal $=, 77$. El perfil de su distribución fue simétrico, $-1,96 \leq \mathrm{Z}_{\mathrm{Sk}}=0,97 \leq 1,96$, y mesocúrtico, $-1,96 \leq \mathrm{Z}_{\mathrm{K} 3}=$ $0,26 \leq 1,96$, y se ajustó a la normalidad por la prueba de D’Agostino y Pearson, $K^{2}$ $=1,02, \mathrm{p}=, 601$. Su media aritmética o promedio fue 3,65 $(<4,5)$, lo que refleja una actitud de aceptación hacia PVVS (1 a 4,49 aceptación versus 4,5 a 9 rechazo).

La consistencia interna de los 20 ítems fue excelente, $\alpha$ ordinal = ,94. La distribución de la ATLG unimodal y con perfil acampanado mostró ligera asimetría positiva, ZSk $=2,63>1,96$, pero fue mesocúrtica, $-1,96 \leq Z_{\mathrm{K} 3}=0,05 \leq 1,96$, pudiéndose mantener la hipótesis nula de normalidad por la prueba de D’Agostino y Pearson con un nivel de significación mayor que $, 01, \mathrm{~K}^{2}=7,83, p=, 020$. Su media de $3,02(<4,2)$ reflejó un posicionamiento de aceptación hacia personas homosexuales.

La consistencia interna de los 12 ítems de la RWA-12 o 13 ítems (incluido el nuevo) fue muy baja, a ordinal $=, 54 \mathrm{y}, 58$, respectivamente. No obstante, al reducir la escala a seis ítems (cinco de los seis ítems redactados en sentido liberal [ítems 2, 4, 6,7 y 10] y el nuevo ítem), se obtuvo una consistencia interna aceptable, $\alpha$ ordinal $=, 79$. La inclusión de cualquier otro ítem disminuía la consistencia interna a un nivel cuestionable. Precisamente, los ítems eliminados presentaban propiedades de discriminabilidad y consistencia interna muy pobres y corresponden a contenidos de agresión autoritaria. La estructura de la RWA reducida a seis ítems (RWA-6) fue de un factor por el análisis paralelo de Horn y la media mínima de las correlaciones parciales al cuadrado. Al extraer el factor desde la matriz de correlación policórica por el método de Residuos Mínimos, se explicó el 39,1\% de la varianza total y las cargas factoriales variaron de ,44 a ,71. Por el contenido de sus ítems, la RWA-6 evalúa autoritarismo desde un rasgo de sumisión a la autoridad y a las normas sociales tradicionales (convencionalismo). La distribución unimodal de la RWA6 fue mesocúrtica, $-1,96 \leq \mathrm{Z}_{\mathrm{K} 3}=0,97 \leq 1,96$, pero presentó asimetría positiva, $\mathrm{Z}_{\mathrm{sk}}=3,83>1,96$, y no se ajustó a una distribución normal. Las puntuaciones por encima de la mediana o valor central estaban más dispersas que las puntuaciones por debajo. No obstante, su media reflejó un posicionamiento liberal, $M=2,74$ ( $<$ 
4,2). Por tanto, unos pocos participantes mostraron mucha sumisión autoritaria y convencionalismo en un grupo de tendencia liberal.

\section{Correlatos con actitud hacia PVVS y hacerse la prueba del VIH}

La EA-PVVS-6 fue independiente de la edad, ser sexualmente activo y haber tratado a PVVS y religiosidad. Correlacionó con una fuerza de asociación media con ser heterosexual, actitud de aceptación hacia personas homosexuales y tener amistad con PVVS y pequeña con autoritarismo de ala derecha, ser hombre, tener amigos homosexuales, hacerse la prueba del VIH y número de parejas sexuales en los últimos seis meses (Tablas 1 y 2). Una mayor aceptación de PVVS está asociada con ser heterosexual, mayor aceptación de las personas homosexuales, tener amistad con PVVS, mayor sumisión a la autoridad y convencionalismo, ser hombre, tener amigos homosexuales, hacerse la prueba del VIH y mayor número de parejas sexuales.

Hacerse la prueba del VIH presentó una fuerza de asociación fuerte con ser sexualmente activo, $\mathrm{r}_{\mathrm{TC}}(191)=, 68$, IC 95\% [,50; ,86], $W(1)=52,33, p<, 001$, y mayor número de parejas sexuales en los últimos seis meses, $\mathrm{r}_{\mathrm{PC}}(169)=, 53$, IC 95\% [,36; ,70], $W(1)=43,89, \mathrm{p}<, 001$. Su fuerza de asociación fue media con ser hombre, $\mathrm{r}_{\mathrm{TC}}(190)=$ ,36, IC 95\% [,12; ,61], $W(1)=8,62, p=, 003$, y baja con una actitud de menor rechazo hacia PVVS, $\mathrm{r}_{\mathrm{PS}}(199)=-, 25,[-0,45,-0,05], W(1)=5,99, \mathrm{p}=, 014$. Fue independiente de la edad, religiosidad, orientación sexual, tratar a PVVS, tener amigos homosexuales o con VIH/SIDA, actitud hacia personas homosexuales y autoritarismo.

Tabla 1.

Correlaciones de las variables cualitativas dicotómicas y ordinales con la EAPVVS-6 y contraste de la normalidad bivariada

\begin{tabular}{|c|c|c|c|c|c|c|c|c|}
\hline \multirow{2}{*}{ Variable } & \multirow{2}{*}{$\mathbf{N}$} & \multirow{2}{*}{$\frac{\mathbf{r}_{\mathrm{PS}}}{(\mathrm{IC} 95 \%)}$} & \multirow{2}{*}{$p$} & \multicolumn{5}{|c|}{ Normalidad bivariada } \\
\hline & & & & $x^{2}$ & $g l$ & $p$ & RMSEA & $p$-close \\
\hline $\begin{array}{l}\text { Sexo }(0=\text { mujer y } 1= \\
\text { hombre })\end{array}$ & 190 & $\begin{array}{r}, 202 \\
(, 030,0,374)\end{array}$ & $<, 001$ & 2,629 & 1 & ,105 & 0,093 & 405 \\
\hline Religiosidad & 197 & $\begin{array}{r}, 101 \\
(-, 040,, 242)\end{array}$ & ,242 & 13,931 & 17 & ,672 & 0 & 1 \\
\hline $\begin{array}{l}\text { Vida sexual activa }(0=\text { no } \\
\text { y } 1=\text { sí })\end{array}$ & 191 & $\begin{array}{r}, 020 \\
(-, 158,, 198)\end{array}$ & ,826 & 0,058 & 1 & ,058 & $<0,001$ & 925 \\
\hline $\begin{array}{l}\text { Orientación heterosexual } \\
(0=\text { no y } 1=\text { sí })\end{array}$ & 198 & $\begin{array}{r}, 430 \\
(, 177,, 683)\end{array}$ & ,001 & 0,030 & 1 & ,863 & $<0,001$ & ,949 \\
\hline $\begin{array}{l}\text { Prueba del HIV }(0=\text { no y } \\
1=\text { sí })\end{array}$ & 199 & $\begin{array}{r}-, 252 \\
(-, 454,-, 050)\end{array}$ &, 014 & 0,410 & 1 &, 522 & $<0,001$ & 800 \\
\hline $\begin{array}{l}\text { Tratar a PVVS }(0=\text { no y } \\
1=\text { sí })\end{array}$ & 173 & $\begin{array}{r}-, 116 \\
(-, 361,, 129)\end{array}$ & ,353 & 0,006 & 1 & ,939 & $<0,001$ & 974 \\
\hline $\begin{array}{l}\text { Amigos homosexuales }(0= \\
\text { no y } 1=\text { sí })\end{array}$ & 191 & $\begin{array}{r}-, 188 \\
(-, 374,-, 002)\end{array}$ &, 048 & 2,432 & 1 & ,119 & 0,087 & ,431 \\
\hline $\begin{array}{l}\text { Amistad con PVVS }(0=\text { no } \\
\text { y } 1=\text { sí })\end{array}$ & 167 & $\begin{array}{r}-, 383 \\
(-, 716,-, 050)\end{array}$ &, 024 & 0,038 & 1 & ,845 & $<0,001$ & ,932 \\
\hline $\begin{array}{l}\text { Nota. } N=\text { número de datos } \\
\text { lo de distribución normal). } \\
\text { del supuesto de normalidad } \\
=\text { valor de probabilidad en } \\
=\text { probabilidad para la } \mathrm{H}_{0}: \mathrm{R}\end{array}$ & $\begin{array}{l}\text { eba } \\
\text { aria } \\
\text { ont }\end{array}$ & $\begin{array}{l}\mathrm{OS}, \mathrm{r}_{\mathrm{PS}}=\text { correla } \\
\text { ignificación: } p \\
\chi^{2}=\text { estadístic } \\
\mathrm{e} \text { a una cola, } \mathrm{R} \\
5 \text { en un contra }\end{array}$ & $\begin{array}{l}\text { ón pol } \\
\text { valor } \\
\text { e la pr } \\
\text { EA = }\end{array}$ & $\begin{array}{l}\text { al, IC } \\
\text { robabi } \\
\text { a de cl } \\
\text { r cuad } \\
\text { Fue }\end{array}$ & & $\begin{array}{l}\text { la pr } \\
\text { do, } g \\
\text { dio }\end{array}$ & $\begin{array}{l}\text { ianza al } \\
\text { de Walc } \\
\text { rados de } \\
\text { roximac }\end{array}$ & $\begin{array}{l}\text { (mode- } \\
\text { ontraste } \\
\text { rtad, } p \\
\text { p-close }\end{array}$ \\
\hline
\end{tabular}


Tabla 2.

Correlaciones de las variables numéricas con la EA-PVVS-6 y contraste de la normalidad bivariada

\begin{tabular}{|c|c|c|c|c|c|c|c|}
\hline \multirow{2}{*}{ Variable } & \multirow{2}{*}{$\mathbf{N}$} & \multirow{2}{*}{$\begin{array}{c}\mathbf{r}_{\mathrm{PS}} \\
\text { (IC 95\%) }\end{array}$} & \multirow{2}{*}{$p$} & \multicolumn{4}{|c|}{ Normalidad bivariada } \\
\hline & & & & $U$ & $p$ & $W$ & $p$ \\
\hline Edad & 199 & $\begin{array}{r}, 076 \\
(-, 055,, 204)^{\mathrm{b}}\end{array}$ & ,286 & 13,376 & 001 & 200,185 & $<, 001$ \\
\hline Número de parejas sexuales & 169 & $\begin{array}{r}-, 181 \\
(-, 314,-, 001)^{b}\end{array}$ & ,018 & 495,130 & $<, 001$ & 14954,403 & $<, 001$ \\
\hline ATLG & 148 & $\begin{array}{r}, 421 \\
(, 272,, 493)^{\mathrm{a}}\end{array}$ & $<, 001$ & 5,853 & 054 & 0,421 & 1 \\
\hline RWA-6 & 196 & $\begin{array}{r}, 276 \\
(, 162,, 397)^{\mathrm{b}}\end{array}$ & $<, 001$ & 9,823 & ,007 & 2,163 & ,726 \\
\hline
\end{tabular}

Notas. ATLG = puntuación total en la escala de actitud hacia lesbianas y hombres homosexuales, RWA-6 = puntaje total del autoritarismo de derecha reducido a seis ítems. $\mathrm{N}=$ número de datos emparejados, $r=$ coeficiente de correlación producto-momento de Pearson, IC = intervalo de confianza al 95\%: ${ }^{a}$ calculado con la distribución t con $n-2$ grados de libertad, ${ }^{b}$ calculado por muestreo repetitivo simple con la simulación de 2000 muestras aleatorias por el método de percentiles; en todos los casos, los valores absolutos de sesgo fueron $\leq, 001$. Prueba de significación: $p=$ valor de probabilidad por la prueba $\mathrm{T}$ en un contraste a dos colas. Pruebas de normalidad multivariada basada en la asimetría (estadístico U) y la curtosis (estadístico W), $p=$ valor de probabilidad. Fuente: elaborada por los autores.

\section{Contraste del modelo predictivo}

Al contrastar el modelo de un factor con seis indicadores de la EA-PVVS-6 (Figura 1), la varianza media extraída fue ,36 con pesos de medida estandarizados de ,50 a ,70, la confiabilidad compuesta de, 77 y el ajuste fue bueno por todos los índices: $\chi 2 / g l=1,10, G F I=, 99, A G F I=, 98, N F I=, 97, R F I=, 96, C F I=, 99, R M S E A=, 03, \mathrm{IC}$ $90 \%[0 ;, 05]$ y $S R M R=, 05$. Por tanto, el modelo de un factor para la escala de actitud hacia PVVS se validó en la muestra.

Al contrastar el modelo de un factor con los seis indicadores de la RWA-6 (Figura 2), la varianza media extraída fue de, 40 con un solo peso de medida estandarizado menor que ,50, $\lambda=, 46$, y la confiabilidad compuesta fue de 79 . El ajuste varió de bueno, $G F I=, 98, A G F I=, 95, N F I=, 95$ y $C F I=, 97$, a aceptable, $\chi 2 / g l=2,41, R F I=$ ,92, $R M S E A=, 09$, IC 90\% [,07; ,10] y $S R M R=, 07$. Por tanto, el modelo de un factor de sumisión a la autoridad y las normas sociales tradicionales para la RWA reducida a 6 ítems mostró una validez aceptable.

Conforme a las conclusiones del estudio de validación de Moral y Valle (2013), se contrastó un modelo de un factor general de homonegatividad para los 20 ítems de la ATLG (Figura 3). Sólo un peso de medida fue menor que ,50, $\lambda=, 49$, la varianza media extraída fue de ,48, la confiabilidad compuesta de 95 y el ajuste varió de bueno, $\chi 2 / g l=1,14, G F I=, 98, A G F I=, 97, N F I=, 97, R F I=, 97, C F I=, 99, R M S E A=, 03$, IC al $90 \%[0 ;, 05]$ a aceptable, $S R M R=, 08$. También el análisis paralelo de Horn y la media mínima de las correlaciones al cuadrado confirmaron un único factor subyacente a la covarianza entre los 20 ítems cuando los modelos de dos o tres factores correlacionados carecieron de validez discriminante entre los factores. Por tanto, el modelo de un factor para la escala de actitud hacia personas homosexuales se validó en la muestra. 


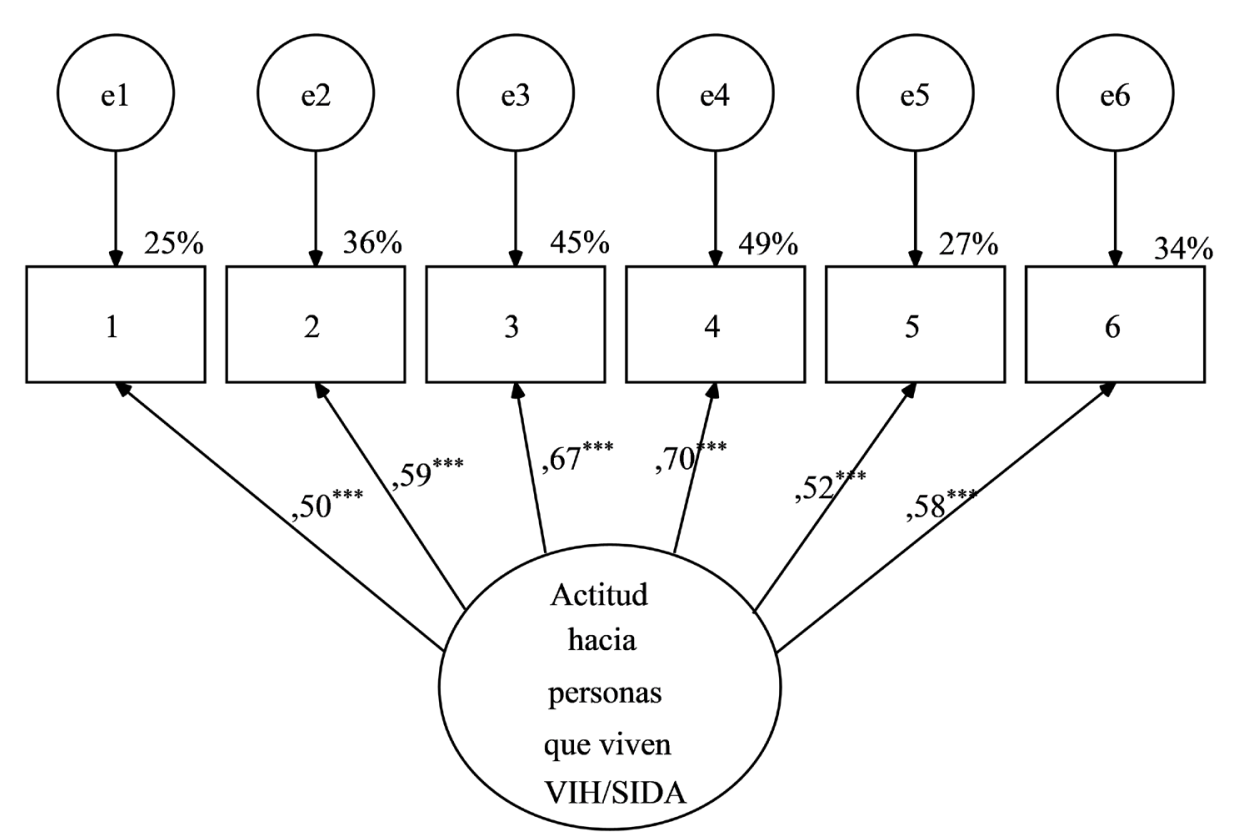

Figura 1. Modelo de un factor con seis indicadores para la EA-PVVS-6 estimado por mínimos cuadrados libres de escala desde la matriz de correlación policórica. Contraste a dos colas por percentiles corregido de sesgo con la simulación de 2000 muestras: ${ }^{* * *} p<, 001 . N=199$. Items de la EA-PVVS-6: 1. Mi actitud hacia las personas con VIH/SIDA es muy positiva. 2. Me gustaría participar en campañas por los derechos de las personas con VIH/SIDA. 3. Es difícil tener a una persona con

$V I H / S I D A$ como amigo cercano. 4. Yo tendría miedo de tocar a una persona con $V I H / S I D A "$. 5. No tendría ningún problema en compartir un departamento con una persona con VIH/SIDA. 6. No me gustaría tener contacto físico con una persona con SIDA. Los ítems 1, 2 y 5 se puntúan en sentido inverso, así todos los items están en sentido autoritario. Fuente: elaborada por los autores.

Para especificar el modelo, se partió de las hipótesis formuladas y se afinaron con los correlatos significativos. Tener amistad con PVVS no se incluyó porque generaba una solución no admisible y reducía el tamaño de la muestra a 108 casos. El número de parejas sexuales tampoco se incluyó como predictor de hacerse la prueba del VIH porque reducía el tamaño de la muestra a 104 casos, generaba que orientación sexual no tuviera pesos significativos y generaba un modelo con mal ajuste y menor potencia explicativa. Se especificó un modelo recursivo (con todas sus relaciones predictivas unidireccionales: $\mathrm{X} \rightarrow \mathrm{Y}$ ) con tres variables exógenas o predictoras (sexo, orientación heterosexual y autoritarismo) y cuatro endógenas o predichas (tener amigos homosexuales, actitud hacia personas homosexuales, actitud hacia PVVS y hacerse la prueba del VIH).

El modelo inicial contó con 17 vías direccionales, tres correlaciones (relaciones no direccionales) entre las tres variables exógenas y cuatro errores estructurales independientes (Figura 4). Contó con cinco participantes por cada parámetro a estimar, $130: 27 \approx 5: 1$, lo que se considera un mínimo aceptable para este tipo de 


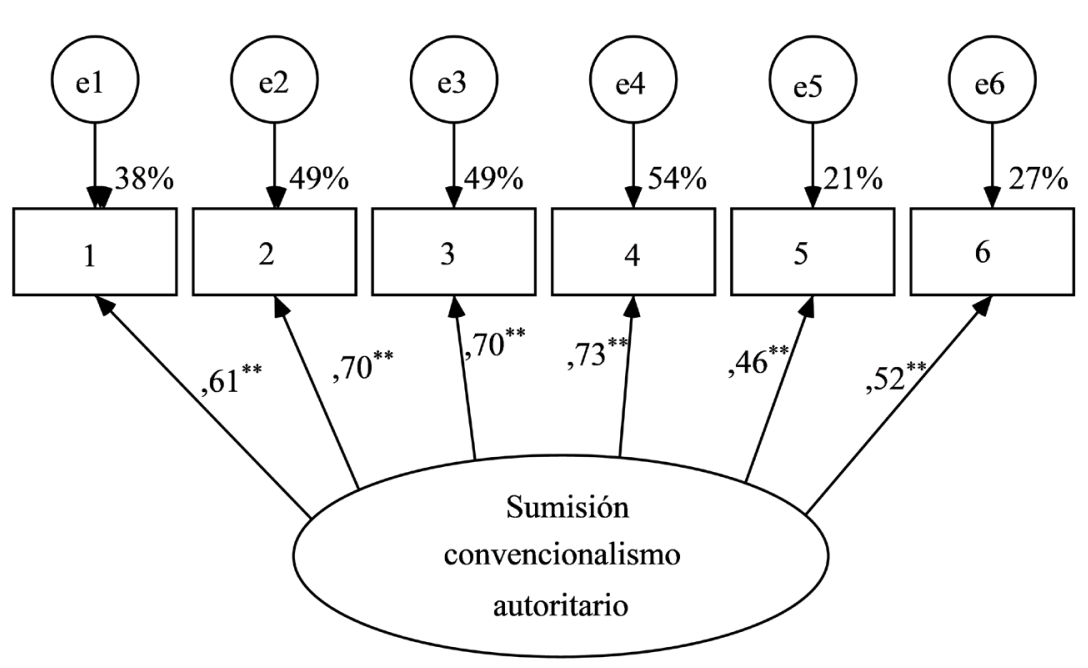

Figura 2. Modelo de un factor con seis indicadores para la RWA-6 (sumisiónconvencionalismo autoritario) estimado por mínimos cuadrados libres de escala desde la matriz de correlación policórica. Contraste a dos colas por percentiles corregido de sesgo con la simulación de 2000 muestras: ${ }^{* *} p<, 01 . N=198$. Items de la RWA-6: 1. Nuestro sociedad necesita libres pensadores, que tengan la valentía para confrontar

los convencionalismos, incluso si esto molestase a muchas personas. 2. Nuestra sociedad sería mejor si mostráramos tolerancia y comprensión por las ideas y valores diferentes (no convencionales). 3. La sociedad necesita mostrar una mayor apertura hacia las personas que piensan por sí mismas y diferente de las autoridades, más que apoyar el que dichas autoridades decidan por nosotros. 4. Muchas personas desafían al Estado, critican a la Iglesia e ignoran las formas normales de vida, sin que por ello dejen de ser buenas. 5. En defensa de la libertad de expresión, deberíamos permitir la publicación de literatura que incluso podemos considerar mala o contraria a nuestras ideas. 6. A una persona abiertamente homosexual se le debería permitir dar servicio en el ejército. Fuente: elaborada por los autores.

análisis (Jöreskog, Olsson y Wallentin, 2016). La solución fue admisible. Cuatro pesos estructurales y dos correlaciones no fueron significativos. Al eliminar estas relaciones no significativas, el modelo final quedó definido por 13 vías direccionales, una correlación entre dos variables exógenas y cuatro residuos estructurales independientes, por lo que se contó con más de seis participantes por cada parámetro a estimar, 130:21 $\approx 6: 1$ (Figura 5).

Una actitud de mayor rechazo hacia PVVS fue predicha por una orientación heterosexual con un tamaño del efecto grande, $\beta=, 52$, ser hombre con un tamaño del efecto medio, $\beta=, 32$, y mayor autoritarismo con un tamaño del efecto pequeño, $\beta=, 18$. Estas tres variables explicaron $45 \%$ de la varianza de la actitud hacia PVVS (Figura 5). La actitud hacia personas homosexuales y tener amigos homosexuales no tuvieron un peso significativo sobre la actitud hacia PVVS, por lo que se eliminaron estas dos vías direccionales inicialmente especificadas. Cabe señalar que al parcializar la varianza de las tres variables predictoras de EA- PVVS-6, la correlación entre 


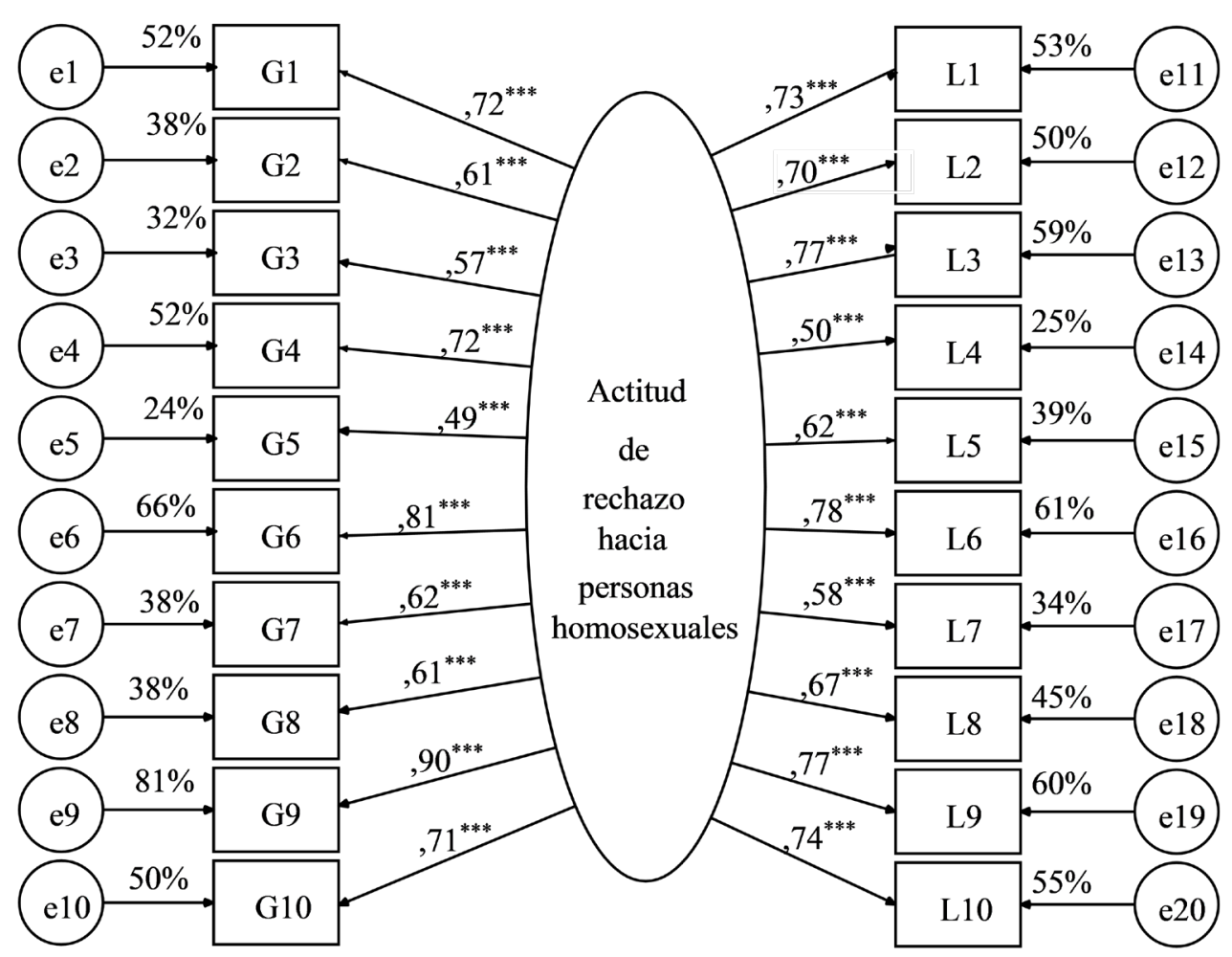

Figura 3. Modelo de un factor con 20 indicadores para la ATLG estimado por mínimos cuadrados libres de escala desde la matriz de correlación policórica.

Contraste a dos colas por percentiles corregido de sesgo con la simulación de 2000 muestras: ${ }^{* * *} p<, 001 . N=149$. Fuente: elaborada por los autores.

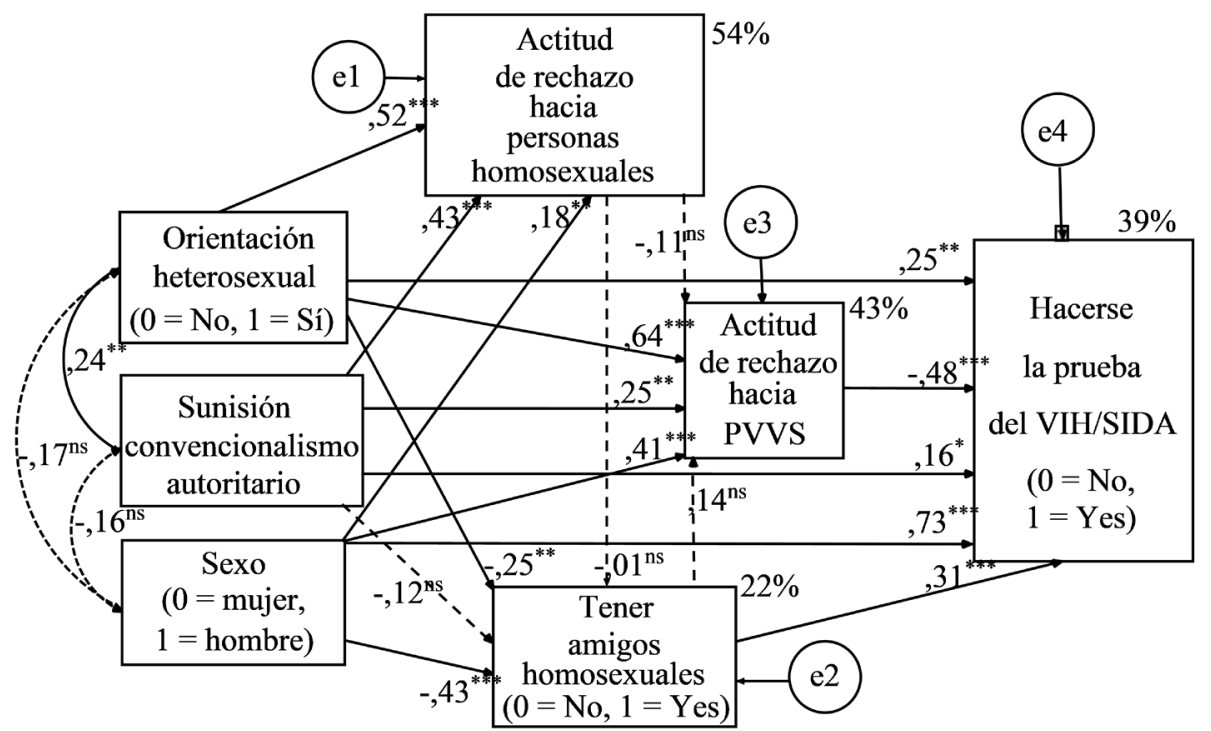

Figura 4. Modelo hipotetizado (excluida la variable número de parejas sexuales) estimado por máxima verosimilitud desde la matriz de correlaciones heterogéneas. $N$ $=130$. Significación en un contraste a dos colas por percentiles corregidos de sesgo con la simulación de 2000 muestras: $n s p>, 05,{ }^{*} p \leq, 05,{ }^{* *} p \leq, 01,{ }^{* *} p \leq, 001$. Fuente: elaborada por los autores. 
ATLG y EA- PVVS-6 disminuyó de ,38 a ,21, pero siguió siendo significativa, $p=$ ,017. No obstante, la correlación bivariada entre amigos homosexuales y EA-PVVS-6 no fue significativa, $\mathrm{r}_{\mathrm{PS}}(130)=-, 15$, IC 95\% $[-, 39 ;, 09], W(1)=1,44, p=0,230$, entre los 130 estudiantes.

La variable endógena hacerse la prueba del VIH fue predicha por ser hombre con un tamaño del efecto muy grande, $\beta=, 72$, una actitud de menor rechazo hacia PVVS y tener amigos homosexuales con tamaños del efecto medios, $\beta=-, 49$ y ,31, respectivamente, y ser heterosexual y mayor autoritarismo con tamaños del efecto pequeños, $\beta=, 25 \mathrm{y}, 16$, respectivamente. Estas cuatro variables explicaron el $41 \% \mathrm{de}$ la varianza de hacerse la prueba del VIH (Figura 5).

Una actitud de mayor rechazo hacia las personas homosexuales fue predicha por una orientación heterosexual con un tamaño del efecto grande, $\beta=, 51$, mayor autoritarismo con un tamaño del efecto medio, $\beta=, 42$, y ser hombre con un tamaño del efecto pequeño, $\beta=, 17$. Estas tres variables explicaron el $57 \%$ de la actitud hacia personas homosexuales (Figura 5).

La cuarta variable endógena fue tener amigos homosexuales. Fue predicha por ser mujer con un tamaño del efecto medio, $\beta=-, 42$, y orientación no heterosexual con un tamaño del efecto pequeño, $\beta=-, 27$. Estas dos variables explicaron el $25 \%$ de la varianza del tener amigos homosexuales (Figura 5). Se eliminó la predicción de tener amigos homosexuales por la actitud hacia personas homosexuales, vía direccional inicialmente especificada, al carecer de peso significativo. Aunque la correlación entre tener amigos homosexuales y la ATLG sí fue significativa, $\mathrm{r}_{\mathrm{PC}}(130)=-, 23$, IC $95 \%[-, 45 ;-, 01], W(1)=4,07, p=, 044$, entre los 130 estudiantes.

De las tres correlaciones entre las tres variables exógenas, sólo la correlación entre orientación sexual y autoritarismo fue significativa. Un mayor autoritarismo presentó una correlación con una fuerza de asociación pequeña con orientación heterosexual, $r=, 24$. Las otras dos correlaciones se eliminaron del modelo, por lo que el sexo quedó independiente de la orientación sexual y el autoritarismo (Figura 5).

El ajuste del modelo fue bueno por siete índices, $\chi 2(7, \mathrm{~N}=130)=13,95, p=, 052$, $p$ de Bollen y Stine $=, 065, G F I=, 971, A G F I=, 88, N F I=, 95, C F I=, 97$ y $R M S E A$ $=, 09$, IC $95 \%[0 ;, 15], p$-close $=, 156, y$ aceptable por $S R M R=, 08$. Además, no mostró sobreparametrización, $\chi 2 / d f=1,99>1$. Esto es una cualidad positiva, ya que un modelo sobreparametrizado logra un ajuste perfecto al tener un exceso de parámetros a estimar que lo aproximan a un modelo saturado, el cual incluye todas las posibles relaciones entre las variables (Jöreskog et al., 2016).

\section{Discusión}

Se formuló como objetivo comprobar un modelo psicosocial para predecir la actitud hacia PVVS y hacerse la prueba del VIH a través de variables sociodemográficas, de religiosidad, conducta sexual, relaciones sociales de amistad, actitud hacia personas homosexuales y autoritarismo de ala derecha. 


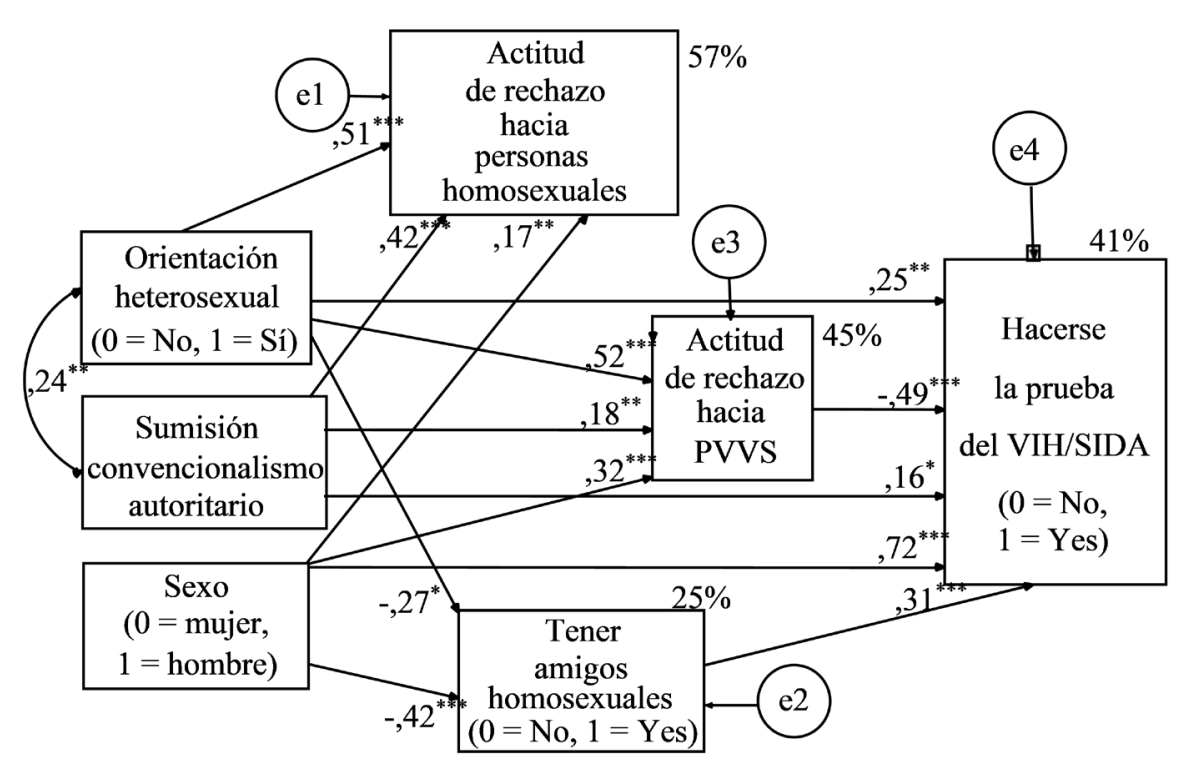

Figura 5. Modelo corregido (sin vías no significativas) estimado por máxima verosimilitud desde la matriz de correlaciones heterogéneas. $N=130$. Significación en un contraste a dos colas por percentiles corregidos de sesgo con la simulación de 2000 muestras: ${ }^{*} p \leq, 05,{ }^{* *} p \leq, 01,{ }^{* * *} p \leq, 001$. Fuente: elaborada por los autores.

Conforme a la expectativa, una actitud de mayor rechazo hacia PVVS fue predicha con un tamaño del efecto grande por orientación heterosexual, ser hombre, así como mayor sumisión a la autoridad y las normas sociales tradicionales. Aunque la actitud hacia personas homosexuales presentó una correlación significativa, su peso estructural en el modelo no fue significativo, por lo que se eliminó esta vía direccional. En la especificación del modelo, la actitud hacia personas homosexuales tuvo los mismos predictores que la actitud hacia PVVS. Al presentar estos tres predictores un tamaño del efecto muy grande sobre la misma, resultó una variable espuria (sin efecto) para predecir actitud hacia PVVS. No obstante, hay que remarcar que la relación entre las dos actitudes es sustantiva a nivel bivariado, ya que la correlación sigue siendo significativa y moderada, al parcializar la varianza de estas tres variables, como también sostienen otros estudios (Emlet et al., 2015).

En la muestra de 130 estudiantes en la que se contrastó el modelo, tener amigos homosexuales fue independiente de la actitud hacia PVVS. Consecuentemente, no tuvo un peso estructural significativo y se eliminó esta vía direccional. La asociación más estable o sustantiva de la actitud hacia PVVS con las dos variables de amistad fue tener amigos que viven con VIH/SIDA. Precisamente, es la que se relaciona directamente con el objeto temido o estigmatizado. No obstante, esta variable no se incluyó en el modelo porque reducía aún más el tamaño de la muestra y generaba una solución no admisible.

Conforme a las expectativas, hacerse la prueba del VIH fue predicho con un tamaño del efecto grande por ser hombre, actitud de mayor aceptación hacia PVVS, tener 
amigos homosexuales y mayor autoritarismo. La orientación sexual también fue un predictor significativo, pero contrario a la expectativa definirse como heterosexual aumenta la probabilidad de hacerse la prueba del VIH. Se esperaba que definirse como no heterosexual fuese la categoría que aumentase la probabilidad (Huerga et al., 2016; Jin et al., 2002), ya que es uno de los grupos de riesgo de infección de VIH/SIDA (Rangel, 2015). Ser heterosexual está relacionado con autoritarismo y es predictor de mayor rechazo hacia PVVS y no tener amigos homosexuales. Así, parece que el discurso de las autoridades de salud contra el negacionismo del VIH/SIDA y a favor de realizarse la prueba del VIH para la detección y tratamiento temprano del VIH/SIDA está más arraigado en este grupo de estudiantes (Kalichman, 2015).

Tener amigos homosexuales se especificó como una variable endógena. Fue predicha con un tamaño del efecto grande por definirse como no heterosexual y ser mujer en conformidad con los estudios de Herek $(1994,2009)$. En la muestra en la que se contrastó el modelo, una actitud de mayor rechazo hacia personas homosexuales correlacionó significativamente con no tener amigos homosexuales. No obstante, tener amigos homosexuales fue independiente de sumisión a la autoridad y las normas sociales tradicionales. ¿Por qué esta faceta del autoritarismo no predijo tener amigos homosexuales y la correlación entre estas dos variables no fue significativa? Puede comprenderse desde el ambiente ideológico y de valores dominantes entre estos estudiantes. Los estudiantes en su mayoría se definieron como heterosexuales (19 de cada 20), sus actitudes promedios fueron de aceptación hacia personas homosexuales y PVVS, su posicionamiento promedio ideológico fue liberal, uno de cada diez indicó ser ateo o agnóstico y la frecuencia mediana de asistencia a servicios religiosos fue baja. Con base en los datos obtenidos, en este contexto social de valores, aceptar a una persona no heterosexual como amigo no parece estar afectado por la ideología política ni la religiosidad, pero sí por la orientación sexual y el sexo, e incluso asociarse con una actitud de aceptación hacia las personas homosexuales. Precisamente, Vaughn et al. (2017) señalan que un contexto de valores antiprejuiciosos facilitan la proximidad hacia las personas no heterosexuales. Probablemente, sea este contexto el que esté anulando el efecto del prejuicio autoritario y religioso a la hora de aceptar la amistad con una persona no heterosexual.

Con respecto a los modelos de medida de las dos de actitudes y el autoritarismo se validan los modelos de un factor. Consecuentemente, este estudio apoya la hipótesis de unidimensionalidad para la ATLG (Blackwell y Kiehl, 2008; Moral y Valle, 2013) frente a modelos de dos (Herek, 1984) y tres factores (Moral y Valle, 2011b), así como para la EA-PVVS-6 (Moral y Valle, en prensa) y una versión reducida de la RWA-6 (sumisión a la autoridad y a las normas sociales tradicionales).

Como limitación del estudio cabe señalar que la técnica de muestreo empleada fue no probabilística, por lo que las inferencias deben tomarse con la debida cautela dentro de la población de estudiantes en la que se hizo la investigación. A pesar del tamaño muestral limitado, éste fue suficiente para las técnicas de análisis usadas (correlaciones heterogéneas con errores estándar menores que ,20 con un aceptable 
cumplimiento de la normalidad bivariada y análisis de senderos con errores generados por técnicas de muestreo repetitivo).

Se concluye que, en esta muestra de estudiantes de medicina de una universidad privada del noreste de México, un modelo estructural, con buen ajuste a los datos y una potencia explicativa entre alta y muy alta para cuatro variables endógenas, predice una actitud de mayor rechazo hacia PVVS a través de una orientación heterosexual, ser hombre y mayor sumisión a la autoridad y las normas sociales tradicionales. Además, pronostica una actitud de mayor rechazo hacia personas homosexuales a través de orientación heterosexual, mayor sumisión a la autoridad y convencionalismo, así como ser hombre. A su vez, anticipa tener amigos homosexuales a través de orientación no heterosexual y ser mujer. Finalmente, predice hacerse la prueba del VIH a través de ser hombre, actitud de aceptación hacia PVVS, tener amigos homosexuales y obediencia o sumisión a las autoridades (sanitarias). En este modelo, la actitud hacia personas homosexuales presenta los mismos predictores que la actitud hacia PVVS, teniendo estos predictores un poder explicativo muy grande, por lo que esta variable resulta redundante ante dichos predictores y no tiene un peso significativo sobre la actitud hacia PVVS, a pesar de que la correlación entre las dos actitudes es sustantiva.

Entre las implicaciones de estos datos, se halla la aportación de evidencias de validez concurrente para la escala EA-PVVS-6 al confirmarse su relación con el sexo, orientación sexual, tener amistad con PVVS y personas homosexuales, hacerse la prueba del VIH, número de parejas sexuales, actitud hacia personas homosexuales y sumisión-convencionalismo autoritario. Entre estos estudiantes de primeros años de carrera, la actitud hacia PVVS es independiente de la edad, religiosidad y tratar a PVVS.

Con base en el modelo, se sugiere desarrollar ambientes sociales de aceptación hacia personas no heterosexuales y posicionamientos ideológicos no autoritarios en las instituciones educativas, lo que evitará la discriminación hacia estas minorías sexuales y facilitará una actitud de aceptación hacia PVVS. En cualquier dinámica orientada al cambio actitudinal, la participación de ambos sexos y personas que se definan como no heterosexuales es importante.

Cabe señalar que, desde el inicio de la epidemia del VIH/SIDA, ha existido polémica sobre la fiabilidad de los test diagnósticos del VIH y la justificación de la terapia antirretroviral de por vida, aunque esta polémica se ha sostenido más bien fuera de la medicina (Goodson, 2014). Hacerse la prueba del VIH y seguir el tratamiento en caso de un resultado positivo es lo indicado si se ha demostrado que el VIH es un lentivirus con base en su purificación y aislamiento, si seha registrado bajo los criterios del Comité Internacional de Taxonomía de Virus, si se tiene el genoma completo del retrovirus aislado, si la relación causal del VIH sobre la inmunodeficiencia ha sido comprobada por estudios independientes con los adecuados controles, si se tienen unos test con criterios diagnósticos claramente establecidos y homogéneos en todos los países, si se ha demostrado a través de ensayos clínicos la eficacia del tratamiento antirretroviral para controlar el VIH y detener el progreso hacia la inmunodepresión 
con un costo aceptable de efectos secundarios. ¿Estos son hechos establecidos para el VIH/SIDA? En el caso de que no, hacerse la prueba del VIH y tomar tratamiento antirretroviral de por vida requieren ser discutidos bajo la lógica de una medicina basada en evidencias (De Harven, 2010; Duesberg et al., 2011; Papadopulos, Turner, Page, Papadimitriou y Causer, 2006).

\section{Referencias}

American Psychological Association. (2017). Ethical principles of psychologists and code of conduct. With the 2016 amendment to standard 3.04. Washington: APA

Asaolu, I. O., Gunn, J. K., Center, K. E. Koss, M. P., Iwelunmor, J. I. y Ehiri, J. E. (2016). Predictors of HIV testing among youth in Sub-Saharan Africa: A cross-sectional study. Plos One, 11(10), e0164052. HTTPS://DOI.ORG/10.1371/JOURNAL. PONE.0164052

Bishop, P. A. y Herron, R. L. (2015). Use and misuse of the Likert item responses and other ordinal measures. International Journal of Exercise Science, 8(3), 297-302.

Blackwell, C. W. y Kiehl, E. M. (2008). Homophobia in registered nurses: Impact on LGB youth. Journal of LGBT Youth, 5(4), 28-44. нTTP:// DX.DOI.ORG/10.1080/19361650802222989

Boone, M. R., Stephanie H. Cook, S. H. y Wilson, P. A. (2016). Sexual identity and HIV status influence the relationship between internalized stigma and psychological distress in black gay and bisexual men. AIDS Care, 28(6), 764-770. HTTPS://DOI.OR G/10.1080/09540121.2016.1164801

Burgess, D. J., Hardeman, R. R., Burke, S. E., Cunningham, B. A., Dovidio, J. F., Nelson, D. B., ... van Ryn, M. (2019). Incoming medical students' political orientation affects outcomes related to care of marginalized groups: Results from the medical student CHANGES study. Journal of Health Politics, Policy and Law, 44(1), 113-146. HTTPS://DOI.ORG/10.1215/036168787206755

Burke, S. E., Dovidio, J. F., Przedworski, J. M., Hardeman, R. R., Perry, S. P., Phelan, S. M., ... van Ryn, M. (2015). Do contact and empathy mitigate bias against gay and lesbian people among heterosexual first-year medical students? A report from the medical student CHANGE study. Academic Medicine, 90(5), 645-651. HTTPS://DOI. ORG/10.1097/ACM.0000000000000661
Byrne, B. (2016). Structural equation modelling with AMOS: Basic concepts, applications, and programming (3a ed.). New York: Routledge.

Cárdenas, M. y Parra, L. (2010). Adaptación y validación de la Versión Abreviada de la Escala de Autoritarismos de Derechas (RWA) en una muestra chilena. Revista de Psicología, 19(1), 61-79. HTTPS://DOI.ORG/10.5354/07190581.2010 .17098

Cui, J., Li, C., Yang, K. y Xu, W. (2018). Adaptive positive and negative runs test. Journal of Statistical Computation and Simulation, 88(7), 1314-1335. HTTPS://DOI.ORG/10.1080/00949655.2 018.1430802

Dahlui, M., Azahar, N., Bulgiba, A., Zaki, R., Oche, O. M., Adekunjo, F. O. y Chinna, K. (2015). HIV/AIDS related stigma and discrimination against PLWHA in Nigerian population. PloS One, 10(12), e0143749. HTtPs://DOI.ORG/10.1371/ JOURNAL.PONE.0143749

Daniel-Ulloa, J., Ulibarri, M., Baquero, B., Sleeth, C., Harig, H. y Rhodes, S. D. (2016). Behavioral HIV prevention interventions among Latinas in the US: A systematic review of the evidence. Journal of Immigrant Minority Health, 18(6), 1498-1521. HTTPS://DOI.ORG/10.1007/S10903-015-0283-0

De Harven, E. (2010). Human endogenous retroviruses and AIDS research: confusion, consensus, or science? Journal of American Physicians and Surgeons, 15(3), 69-74.

Duesberg, P. H., Mandrioli, D., McCormack, A., Nicholson, J. M., Rasnick, D., Fiala, C., Koehnlein, C., Bauer, H. H. y Ruggiero, M. (2011). AIDS since 1984: No evidence for a new, viral epidemic - not even in Africa. Italian Journal of Anatomy and Embryology, 116(2), 73-92.

Efron, B. y Hastie, T. (2016). Computer age statistics inference: algorithms, evidence and data science. New York: Cambridge University Press. 
Emlet, C. A., Brennan, D. J., Brennenstuhl, S., Rueda, S., Hart, T. A. y Rourke, S. B. (2015). The impact of HIV-related stigma on older and younger adults living with HIV disease: does age matter? AIDS Care, 27(4), 520-528. HTTPS://DOI.ORG/10.1080/09 540121.2014 .978734

Espinoza, S. C. y Novoa-Muñoz, F. (2018). Advantages of ordinal alpha versus Cronbach's alpha, illustrated using the WHO AUDIT test. Pan American Journal of Public Health, 42, e65. HTTPS://DOI.ORG/10.26633/RPSP.2018.65

Farhat, D., Greene, E., Paige, M. Q., Koblin, B. A. y Frye, V. (2017). Knowledge, stereotyped beliefs and attitudes around HIV chemoprophylaxis in two high HIV prevalence neighborhoods in New York City. AIDS and Behavior, 21(5), 1247-1255. HTTPS://DOI.ORG/10.1007/s10461-016-1426-6

Geter, A., Herron, A. R. y Sutton, M. Y. (2018). HIVrelated stigma by healthcare providers in the United States: A systematic review. AIDS Patient Care and STDs, 32(10), 418-424. HTTPS://DOI. ORG/10.1089/APC.2018.0114

Goodson, P. (2014). Questioning the HIV-AIDS hypothesis: 30 years of dissent. Frontiers in Public Health, 2, 154. HтtPs://Dol.ORG/10.3389/ FPUвн.2014.00154

Herek, G. M. (1984). Attitudes toward lesbians and gay men: A factor analytic study. Journal of Homosexuality, 10(1-2), 39-51. HTTPs://DOI. ORG/10.1300/J082V10NO1_03

Herek, G. M. (1994). Assessing heterosexuals attitudes toward lesbians and gay men: A review of empirical research with the ATLG scale. En B. Greene y G. M. Herek, (Eds.), Lesbian and gay psychology: Theory, research, and clinical applications (p. 206-228). Thousand Oaks: Sage Publications.

Herek, G. M. (1996). Why tell if you're not asked? Selfdisclosure, intergroup contact, and heterosexuals attitudes toward lesbians and gay men. En G. M. Herek, J. B. Jobe y R. M. Carney (Eds.), Worlds of desire. Out in force: Sexual orientation and the military (pp. 197-225). Chicago: University of Chicago Press.

Herek, G. M. (2009). Sexual stigma and sexual prejudice in the United States: A conceptual framework. En D. A. Hope (Ed.), Contemporary perspectives on lesbian, gay, and bisexual identities (pp. 65-111). New York: Springer.

Hodson, G., Turner, R. N. y Choma, B. L. (2016). Individual differences in intergroup contact propensity and prejudice reduction. En L. Vezzali y S. Stathi (Eds.), Intergroup contact theory: Recent developments and future directions (pp. 8-31). London: Routledge.
Huerga, H., Van Cutsem, G., Ben Farhat, J., Reid, M., Bouhenia, M., Maman, D., ... Ellman, T. (2016). Who needs to be targeted for HIV testing and treatment in KwaZulu-Natal? Results from a population-based survey. Journal of Acquired Immune Deficiency Syndromes, 73(4), 411-418. HTTPS://DOI.ORG/10.1097/QAI.0000000000001081

Jin, F., Prestage, G., Law, M., Kippax, S., Van de Ven, P., Rawsthorne, P., ... Grulich, A. (2002). Predictors of recent HIV testing in homosexual men in Australia. HIV Medicine, 3(1), 271-276. нттрs:// DOI.ORG/10.1046/J.1468-1293.2002.00121.X

Jöreskog, K. G., Olsson, U. H. y Wallentin, F. Y. (2016). Multivariate analysis with LISREL. Cham: Springer.

Kalichman, S. C. (2015). Commentary on "questioning the HIV-AIDS hypothesis: 30 years of dissent". Frontiers in Public Health, 3, 1-2, 30. HTTPS://DOI.ORG/10.3389/FPUBH.2015.00030

Kikwasi, G. J., Lukwale, S. R. y Mwageni, E. A. (2017). A study of first year students' attitude towards HIV and AIDS. Global Journal of Health Science, 9(7), 117-126. HTTPs://DOI.ORG/10.5539/GJHs. v9N7P117

Knaak, S y Patten, S. A (2016). Grounded theory model for reducing stigma in health professionals in Canada. Acta Psychiatrica Scandinavica, 134(Suppl. 446), 53-62. HTTPS://DOI.ORG/10.1111/ ACPS. 12612

Kyriazos, T. A. (2018). Applied psychometrics: sample size and sample power considerations in factor analysis (EFA, CFA) and SEM in general. Psychology, 9(8), 2207-2230. HTtPs://DOI. ORG/10.4236/PSYCH.2018.98126

Masoudnia, E. (2015). Public perceptions about HIV/AIDS and discriminatory attitudes toward people living with acquired immunodeficiency syndrome in Iran. Journal of Social Aspects of HIV/AIDS, 12(1), 116-122. HTTPS://DOI.ORG/10.1 080/17290376.2015.1123644

Moral, J. (2019). Revisión de los criterios para validez convergente estimada a través de la varianza media extraída. Psychologia, 13(2), 25-41.

Moral, J. y Valle, A. (2011a). Escala de Actitudes hacia Lesbianas y Hombres Homosexuales (ATLG) 2. Distribución y evidencias de validez. Revista Electrónica Nova Scientia, 4(7), 153-171. нттрs:// DOI.ORG/10.21640/NS.V4I7.180

Moral, J. y Valle, A. (2011b). Escala de Actitudes hacia Lesbianas y Hombres Homosexuales en México 1. Estructura factorial y consistencia interna. Revista Electrónica Nova Scientia, 3(6), 139-157. HTTPS://DOI.ORG/10.21640/NS.V316.191 
Moral, J. y Valle, A. (2013). Contraste de una estructura trifactorial para escala de actitud hacia lesbianas y hombres homosexuales (ATLG). PSICUMEX, 3(2), 33-51.

Moral, J. y Valle, A. (2019). Concurrent validity of the internalized homonegativity scale in its versions composed of 14 and 16 items. En C. H. García (Ed.), Researches in Hispanic Psychology (Vol., 2, pp. 97-146). Hauppauge: Nova Science.

Moral, J. y Valle, A. (en prensa). Propiedades psicométricas de la Escala de Actitud hacia Personas que Viven con VIH/SIDA. Perspectivas Sociales, 21(2).

Mukherjee, S., McKinney, S. y Darrow, W. (2018). Stigma towards homosexuality and AIDS among students of a large Hispanic-serving university. Sexuality \& Culture, 22(4), 1137-1153. нттPs:// DOI.ORG/10.1007/S12119-018-9516-4

Neumann, R., Hülsenbeck, K. y Seibt, B. (2004). Attitudes towards people with AIDS and avoidance behavior: Automatic and reflective bases of behavior. Journal of Experimental Social Psychology, 40(4), 543-550. HTTPS://DOI. ORG/10.1016/J.JESP.2003.10.006

Papadopulos, E., Turner, V. F., Page, B. A., Papadimitriou, J. y Causer, D. (2006). No proof HIV antibodies are caused by a retroviral infection. Emergency Medicine Australas, 18(3), 308-309. HTTPS://DOI.ORG/10.1111/J.1742$6723.2006 .00859 . \mathrm{x}$

Prati, G., Zani, B., Pietrantoni, L., Scudiero, D., Perone, P., Cosmaro, L., ... Oldrini, M. (2016). The role of knowing someone living with HIV/ AIDS and HIV disclosure in the HIV stigma framework: a Bayesian mediation analysis. Quality \& Quantity, 50(2), 637-651. HTTPs://DOI. ORG/10.1007/S11135-015-0168-2

Rangel, Y. Y. (2015). Narrativas del riesgo respecto del VIH/sida en México. De letal a crónica y del estigma a los derechos humanos. Revista de El Colegio de San Luis, 5(9), 200-219. HTTPs://DOI. ORG/10.21696/RCSL592015612

Reyes, M., Varas, N. y Martínez, M. T. (2015) Religion and HIV/AIDS stigma: Considerations for the nursing profession. The New School Psychology Bulletin, 12(1), 48-55.
Rickles, N. M., Furtek, K. J., Malladi, R., Ng, E. y Zhou, M. (2016). Pharmacy student attitudes and willingness to engage in care with people living with HIV/AIDS. American Journal of Pharmaceutical Education, 80(3), artículo 45. HTTPS://DOI.ORG/10.5688/AJPE80345

Suominen, T., Laakkonen, L., Lioznov, D., Polukova, M., Nikolaenko, S., Lipiäinen, L., ... Kylmä, J. (2015). Russian nursing students' knowledge level and attitudes in the context of human immunodeficiency virus (HIV) - a descriptive study. BMC Nursing, 14(1), 1-12. HTTPs://DoI. ORG/10.1186/s12912-014-0053-7

Tang, W., Zhuang, X., Zhao, H., Pang, C., He, Y., Liu, F., ... He, D. (2016). HIV/AIDs-related stigma among medical students in Beijing, China. International Journal of Clinical and Experimental Medicine, 9(5), 8743-8748.

Ungaretti, J., Müller, M. y Etchezahar, E. (2016). El estudio psicológico del prejuicio: Aportes del autoritarismo y la dominancia social. Revista Internacional de Investigación en Ciencias Sociales, 12(1), 75-86. HTTPS://DOI.ORG/10.18004/ RIICS.2016.JULIO.75-86

Vaughn, A. A, Teeters, S. A., Sadler, M. S. y Cronan, S. B. (2017). Stereotypes, emotions, and behaviors toward lesbians, gay men, bisexual women, and bisexual men. Journal of Homosexuality, 64(13), 1890-1911. HTTPS://DOI.ORG/10.1080/00918369.20 16.1273718

Weiser, S. D., Heisler, M., Leiter, K., Percy-de Korte, F., Tlou, S., DeMonner, S., ... Bangsberg, D. R. (2006). Correction: Routine HIV testing in Botswana: A population-based study on attitudes, practices, and human rights concerns. PLOS Medicine, 3(10), e395. HTTPs://DOI.ORG/10.1371/ JOURNAL.PMED.0030395

Wesolowski, L., Chavez, P., Sullivan, P., Freeman, A, Sharma, A., Mustanski, B., ... MacGowan, R. (2019). Distribution of HIV self-tests by HIVpositive men who have sex with men to social and sexual contacts. AIDS and Behavior, 23(4), 893899. HTTPS://DOI.ORG/10.1007/S10461-018-2277-0 